\title{
Low-Lying Excited States of Quantum Antiferromagnets \\ on a Triangular Lattice円
}

\section{Tsutomu Momoi}

Department of Physics, University of Tokyo, Hongo 7-3-1, Bunkyo-ku, Tokyo 113, Japan

\begin{abstract}
We study low-lying states of the $X Y$ and Heisenberg antiferromagnets on a triangular lattice to clarify whether spontaneous symmetry breaking occurs at $T=0$ in the thermodynamic limit. Approximate forms of low-lying states are proposed, in which degrees of freedom of the sublattice magnetization and of the chirality are separated. It is shown that low-lying states can be accurately described with the present approximation. It was argued that low-lying states play an important role in symmetry breaking. With help of this approximation, we discuss the contribution of low-lying states to symmetry breaking of two types, namely creation of the spontaneous sublattice magnetization and the spontaneous chirality. Furthermore, to show an evidence for the occurrence of the symmetry breaking, we numerically study the low-lying states of finite systems of the $X Y$ and Heisenberg antiferromagnets. It is found that the necessary conditions for the symmetry breaking to occur are satisfied in these models.
\end{abstract}

KEY WORDS: Quantum antiferromagnets, Heisenberg model, XY model, triangular lattice, low-lying states, symmetry breaking.

\section{Introduction}

Many authors have studied ground-state properties of quantum antiferromagnets, e.g., the Heisenberg and $X X Z$ models, on a triangular lattice. The Hamiltonian of the $X X Z$ antiferromagnet is given by

$$
H=J \sum_{\langle i, j\rangle}\left(S_{i}^{x} S_{j}^{x}+S_{i}^{y} S_{j}^{y}+\Delta S_{i}^{z} S_{j}^{z}\right)
$$

where the summation runs over all the nearest-neighbour pairs. Most of the studies are based on one of the following two viewpoints. The first one is that the system is disordered. Anderson proposed the RVB state(1, E), and Kalmeyer and Laughlin discussed a spin-liquid state (3) from this aspect. The second one is that the system has a long-range order with the so-called $120^{\circ}$ structure, though quantum fluctuations reduce the sublattice magnetization. Trial wave functions which have the $120^{\circ}$ structure are discussed by Miyashita (4) The spin-wave expansions (6, 月, 8, (9) and the series expansion 10 suggested the existence of a longrange order. The present author and Suzuki presented the exact ground states of the $X X Z$

\footnotetext{
${ }^{1}$ To be published in J. Stat. Phys. 75 (1994)
} 
model with $\Delta=-0.5$, most of which have the $120^{\circ}$ structures. We also studied the $X X Z$ antiferromagnet in the region $-0.5<\Delta \leq 1$ using the spin-wave expansion and showed that the quantum fluctuations are enhanced, as $\Delta$ becomes large. The quantum effect is strong in the Heisenberg model. The estimates of the ground-state energy in the studies based on these pictures are almost equal to each other in the Heisenberg model. Thus it is difficult to judge which picture is correct.

Apart from the above arguments, several authors 11, 12, 96 studied ground states in finite systems using the exact-diagonalization method. There are two papers 12, 6) which reported the calculations up to $N=36$. Conclusions do not coincide with each other. Bernu, Lhuillier and Pierre ${ }^{12}$ ) reported that the sublattice order is almost equal to that obtained by the spinwave expansion. Leung and Runge's result is, however, contradictory to the existence of the sublattice order. These two groups fit the data in different ways.

Understanding of symmetry breaking has progressed by studies of low-lying excited states. In quantum antiferromagnets the ground state of a finite system is, in many cases, unique and symmetric. In the thermodynamic limit, symmetry is broken by making a linear combination of the ground state and low-lying excited states. Horsch and von der Linden(13) first showed that, if the Heisenberg antiferromagnet has a Néel order, there exists a low-lying excited state whose energy converges to that of the ground state. Koma and Tasaki(4) rigorously proved that, if the system has a long-range order, low-lying states of growing numbers converge to the ground state in the thermodynamic limit. Several numerical calculations showed the existence of these low-lying excited states in the spin 1/2 Heisenberg antiferromagnet on a square lattice(15) and on a triangular lattice (12).

Bernu et al. 12 determined the quantum numbers of low-lying states which construct the ordered infinite-volume ground states in the Heisenberg antiferromagnet on the triangular lattice. They studied finite systems and found the existence of a whole set of low-lying states. Azaria et al. (16) studied the finite-size dependence of these low-lying states. They found that these states satisfy the scaling property which indicates the existence of a long-range order.

In this paper we consider the $X Y$ and Heisenberg antiferromagnets on the triangular lattice. We study low-lying states to clarify whether spontaneous symmetry-breaking occurs at $T=0$ in the thermodynamic limit. We give approximate forms of low-lying states, in which the degrees of freedom of the sublattice magnetization and the chirality are separated. We show that the low-lying states can be accurately described with the present approximation. With help of our approximation we discuss how rearrangements of the low-lying states bring on symmetry breaking. In quantum antiferromagnets on the triangular lattice, there are two types of symmetry breaking; In the Heisenberg antiferromagnet both the sublattice magnetization and the chirality relate to the breaking of the $O(3)$ symmetry and in the $X Y$ antiferromagnet the $O(2) \times Z_{2}$ (chiral) symmetry can be broken. These symmetry breakings relate to each other. Using our approximation we can understand breakdown of each symmetry independently. Furthermore, studying the low-lying states of finite systems with the numerical diagonalization 
method, we obtain an evidence of occurrence of the symmetry breaking. In the $X Y$ model the necessary conditions for the symmetry to break are fully satisfied. We conclude that there exists long-range order in the thermodynamic limit. In the Heisenberg model, since the system size studied here is too small, we can not still obtain a definite conclusion about the existence of long-range order, but there are many evidences which support the existence of the long-range order, as discussed in refs. 12 and 16.

The relation between the long-range order parameter $\sigma$ and the spontaneous sublattice magnetization $m$ is also argued, though this is not the main part of the present paper. It has been discussed (17) that, for bipartite systems, the relation is given by $m=\sqrt{3} \sigma$ in the Heisenberg antiferromagnet and by $m=\sqrt{2} \sigma$ in the $X Y$ antiferromagnet. In the antiferromagnets on the triangular lattice, however, the factor of the relation becomes $\sqrt{2}$ times as large as the above. We obtain $m=\sqrt{6} \sigma$ for the Heisenberg model and $m=2 \sigma$ for the $X Y$ model.

Contents of this paper are the following. Approximate low-lying states are formally given and properties of these states are discussed in Section 2. Some results for the low-lying states of ferromagnets, which are used in Section 2 to discuss the properties of the approximation, are shown in Appendix A. The mechanism of symmetry breaking is discussed in Section 3. In Section 4 we show an evidence of the symmetry breaking studying finite systems numerically. Section 5 contains summary and discussions. The relations between the symmetry breaking and the long-range order parameter are discussed in Appendix B.

\section{An approximation for low-lying states}

We study properties of the low-lying states of the quantum antiferromagnets on a finite-volume triangular lattice, using approximate states. In antiferromagnets on finite systems the ground state is symmetric. Symmetry breaking in an infinite-volume limit is discussed in the next section.

We consider the quantum $X X Z$ antiferromagnets on the finite-volume lattice $\Lambda$ with a periodic boundary condition. The size of the system is $N$, where $N=3 m$ with an integer $m$. The Hamiltonian is given by

$$
H=J \sum_{\langle i, j\rangle \in \Lambda}\left(S_{i}^{x} S_{i}^{x}+S_{i}^{y} S_{i}^{y}+\Delta S_{i}^{z} S_{i}^{z}\right)
$$

where the summation runs over all the nearest-neighbor sites and the symbol $\Delta$ denotes the anisotropy of the z-component interactions. The models in the region $-0.5<\Delta \leq 1$ are considered.

We concentrate on low-lying states which have sublattice-translational invariance and $C_{3 V^{-}}$ invariance. (We denote the point group of the $120^{\circ}$ rotation and reflection of the lattice as $C_{3 V}$.) Bernu et al. (12) reported that there are many low-lying states of this type. Here we classify the states according to eigenvalues of the $60^{\circ}$ rotation $\left(C_{6}\right)$. We call the class of $C_{6}$-symmetric states as the type $\alpha$ and the class of $C_{6}$-antisymmetric states as the type $\beta$. 
As an approximation for the lowest states of the type $\alpha$ and of the type $\beta$ in the $S_{\text {total }}^{z}=n$ (or $n+1 / 2$ ) subspace, we consider the following states:

$$
|n \alpha\rangle=\frac{\left(U+U^{\dagger}\right)|\mathrm{F} n\rangle}{\|\left(U+U^{\dagger}\right)|\mathrm{F} n\rangle \|}, \quad|n \beta\rangle=\frac{\left(U-U^{\dagger}\right)|\mathrm{F} n\rangle}{\|\left(U-U^{\dagger}\right)|\mathrm{F} n\rangle \|}
$$

for $n=0, \pm 1, \pm 2, \ldots$, where the symbol $U$ denotes the unitary operator

$$
U=\exp \left(i \frac{2 \pi}{3} \sum_{i \in B} S_{i}^{z}-i \frac{2 \pi}{3} \sum_{i \in C} S_{i}^{z}\right)
$$

and $\|\left(U \pm U^{\dagger}\right)|\mathrm{F} n\rangle \|=\left\langle\mathrm{F} n\left|\left(U \pm U^{\dagger}\right)^{2}\right| \mathrm{F} n\right\rangle^{1 / 2}$. The state $|\mathrm{F} n\rangle$ denotes the lowest state in the $S_{\text {total }}^{z}=n($ or $n+1 / 2)$ subspace of the "ferromagnetic" $X X Z$ model on $\Lambda$ which is defined by the Hamiltonian

$$
H_{\mathrm{F}}=-J \sum_{\langle i, j\rangle \in \Lambda}\left(S_{i}^{x} S_{j}^{x}+S_{i}^{y} S_{j}^{y}-2 \Delta S_{i}^{z} S_{j}^{z}\right)
$$

Here the parameter $\Delta$ is set equal to that in (2.1). We denote the eigenvalue of $H_{F}$ for the state $|\mathrm{F} n\rangle$ as $E_{\mathrm{f} n}$.

We first discuss the properties of the above approximate states (2.2). After that we verify that this approximation is good.

Here we discuss the meaning of the referred model (2.4). The states $|n \alpha\rangle$ and $|n \beta\rangle$ are linear combinations of $U|\mathrm{~F} n\rangle$ and $U^{\dagger}|\mathrm{F} n\rangle$. The unitary operator $U$ transforms the Hamiltonian (2.1) into the form

$$
U H U^{\dagger}=-\frac{1}{2} J \sum_{\langle i, j\rangle}\left(S_{i}^{x} S_{j}^{x}+S_{i}^{y} S_{j}^{y}-2 \Delta S_{i}^{z} S_{j}^{z}\right)-\frac{\sqrt{3}}{2} J \sum_{\langle i \rightarrow j\rangle}\left(S_{i}^{x} S_{j}^{y}-S_{i}^{y} S_{j}^{x}\right) .
$$

Here the symbol $i \rightarrow j$ goes from the sublattice $\mathrm{A}$ to $\mathrm{B}, \mathrm{B}$ to $\mathrm{C}$, and $\mathrm{C}$ to $\mathrm{A}$. The first term in (2.5) gives the Hamiltonian (2.4). Thus the present approximation corresponds to neglecting the second term in (2.5).

In the thermodynamic limit of the ferromagnet (2.4), only $O(2)$ symmetry breaking can take place, since it has no frustration. The classical limit of the ferromagnet (2.4) in the region $-0.5<\Delta \leq 1$ has ferromagnetically ordered ground states, in which all spins are lying in the $X Y$ plane(18). The spin $1 / 2$ ferromagnetic $X Y$ model $(\Delta=0)$ on the triangular lattice has been studied by using the exact-diagonalization method (11) and the spin-wave theory $(10,20)$. The results indicate the existence of the ferromagnetic long-range order. As $\Delta$ increases in the ferromagnet (2.4), quantum fluctuations are enhanced and the magnetization is reduced.

As shown in Appendix A, several exact results can be obtained for the ferromagnet (2.4) on the finite-volume lattice $\Lambda$. The ground state is unique and it exists in the $S_{\text {total }}^{z}=0$ subspace. Then it has $O(2)$-rotational invariance. (When $N$ is an odd number, there exists trivial degeneracy; the states $|\mathrm{F} 0\rangle$ and $|\mathrm{F}-1\rangle$ are degenerate.) The lowest state in the $S_{\text {total }}^{z}=n$ subspace, which we denote as $|\mathrm{F} n\rangle$, is unique. The coefficients of $|\mathrm{F} n\rangle$ in the basis which are eigenstates of $\left\{S_{i}^{z}\right\}$ are nonnegative. All states $\{|\mathrm{F} n\rangle\}$ are translationally invariant and $C_{6 V}$-invariant. (See Appendix A.) 
Table 1: Overlaps between the ground state $|0\rangle$ and the approximate state $|0 \alpha\rangle$, and between the first excited state $|1\rangle$ and the approximate one $|0 \beta\rangle$.

\begin{tabular}{|c|c|c|c|c|c|}
\hline \multicolumn{2}{|c|}{ Size $N$} & 3 & 9 & 12 & 21 \\
\hline \multirow{2}{*}{$X Y$} & $|\langle 0 \mid 0 \alpha\rangle|^{2}$ & 1.0 & 0.9797 & 0.8472 & 0.9181 \\
\hline & $|\langle 1 \mid 0 \beta\rangle|^{2}$ & 1.0 & 0.9797 & 0.9988 & 0.9181 \\
\hline \multirow{2}{*}{ Heisenberg } & $|\langle 0 \mid 0 \alpha\rangle|^{2}$ & 1.0 & 0.7881 & 0.7997 & 0.4643 \\
\hline & $|\langle 1 \mid 0 \beta\rangle|^{2}$ & 1.0 & 0.7881 & 0.9871 & 0.4643 \\
\hline
\end{tabular}

Using these rigorous results for the low-lying states of the ferromagnet, we can determine the spatial quantum numbers of the approximate states. We have verified in the small clusters that the spatial quantum numbers of the approximate states (2.2) are the same as those of the true states. Bernu et al. 122 investigated the spatial quantum numbers of the low-lying states for the Heisenberg antiferromagnet and classified the low-lying states into three classes; $\Gamma_{1}$ (states with $k=0$ and even under inversion), $\Gamma_{2}$ (states with $k=0$ and odd under inversion) and $\Gamma_{3}$ (states with $\left.k= \pm(4 \pi / 3,0)\right)$. When $S_{\text {total }}^{z}(=n)=3 l$ or $S_{\text {total }}^{z}(=n+1 / 2)=3 l+3 / 2$ with an integer $l,|n \alpha\rangle$ and $|n \beta\rangle$ belong to $\Gamma_{1}$ and $\Gamma_{2}$, respectively. Otherwise, both $|n \alpha\rangle$ and $|n \beta\rangle$ belong to $\Gamma_{3}$. As we show in the next section, our classification is useful in discussing the symmetry breaking about the sublattice magnetization and the chirality separately.

We now discuss accuracy of our approximation. We compare the approximate low-lying states with the true ones, numerically diagonalizing finite systems. First we note that, as we have already mentioned above, the spatial quantum numbers of our approximate low-lying states are equal to those of the true states: They are invariant under any sublattice translation and any translation of $C_{3 V}$, and they have the same momentum as the true states. In the true system there exist low-lying states of two types corresponding to the types $\alpha$ and $\beta$. Secondly the approximate low-lying states have accurate expectation values of the energy, which are at most $2 \%$ higher than the exact values in the $X Y$ model and at most $7 \%$ higher in the Heisenberg model. Finally we calculated overlaps between the exact low-lying states and the approximate ones. The results are shown in Table 1. In the $X Y$ model the approximate states have more than $90 \%$ overlaps with the exact states and in the Heisenberg model more than $70 \%$. These situations are the same for the low-lying states in the $S_{\text {total }}^{z}=1$ and $S_{\text {total }}^{z}=2$ subspaces. Thus we find that the exact low-lying spectrum has the two-fold structures, as shown in Fig. 1, and that the true low-lying states can be accurately described with the present approximation.

The approximate ground state, $|0 \alpha\rangle$, is a generalization of the trial state of Betts and Miyashita(21) for the $X Y$ antiferromagnet. In the case of $\Delta=-0.5,|0 \alpha\rangle$ and $|0 \beta\rangle$ belong to the exact ground states, which were presented by the author and Suzuki( $\mathbb{Q}^{2}$. This fact partially gives the reason for accuracy of the state $U|\mathrm{~F} 0\rangle$. 
An advantage of our approximation is that the degrees of freedom of the $Z_{2}$ (chiral) symmetry and the $O(2)$ symmetry are separated in it. Then we can discuss breakdown of each symmetry independently. The parts of the unitary operator, namely $\left(U \pm U^{\dagger}\right)$, can describe the chiral symmetry breaking and the parts of the low-lying states of the ferromagnet, namely $|\mathrm{F} n\rangle$ $(n=0, \pm 1, \pm 2, \ldots)$, can display the breakdown of the $O(2)$ symmetry.

Using this approximation, we can estimate the expectation values of the energy. The groundstate energy is calculated as

$$
\langle 0 \alpha|H| 0 \alpha\rangle=\frac{E_{\mathrm{f} 0}+4 E_{\mathrm{f} 0}\langle\mathrm{~F} 0|U| \mathrm{F} 0\rangle-6 \Delta J\left\langle\mathrm{~F} 0\left|U\left(\sum_{\langle i, j\rangle} S_{i}^{z} S_{j}^{z}\right)\right| \mathrm{F} 0\right\rangle}{2+2\langle\mathrm{~F} 0|U| \mathrm{F} 0\rangle},
$$

where we have used the relations $U H U^{\dagger}+U^{\dagger} H U=H_{\mathrm{F}}$ and $U H U=H_{\mathrm{F}} U^{\dagger}+U^{\dagger} H_{\mathrm{F}}-$ $3 \Delta J U^{\dagger} \sum_{\langle i, j\rangle} S_{i}^{z} S_{j}^{z}$. The terms $\langle\mathrm{F} 0|U| \mathrm{F} 0\rangle$ and $\left\langle\mathrm{F} 0\left|U\left(\sum_{\langle i, j\rangle} S_{i}^{z} S_{j}^{z}\right)\right| \mathrm{F} 0\right\rangle$ correspond to the transition probabilities from the ferromagnetic ground state, $|\mathrm{F} 0\rangle$, to the antiferromagnetic state, $U|\mathrm{~F} 0\rangle$. In the thermodynamic limit, these values are vanishing. Thus the ground-state energy is estimated as

$$
\langle 0 \alpha|H| 0 \alpha\rangle \simeq \frac{E_{\mathrm{f} 0}}{2}
$$

Next we discuss the energy gaps. The energy gap between the ground state (the type $\alpha$ ) and the lowest state of the type $\beta$ is almost vanishing as

$$
\begin{aligned}
\langle 0 \beta|H| 0 \beta\rangle-\langle 0 \alpha|H| 0 \alpha\rangle & =\frac{-3 E_{\mathrm{f} 0}\langle\mathrm{~F} 0|U| \mathrm{F} 0\rangle+6 \Delta J\left\langle\mathrm{~F} 0\left|U\left(\sum_{\langle i, j\rangle} S_{i}^{z} S_{j}^{z}\right)\right| \mathrm{F} 0\right\rangle}{1-\langle\mathrm{F} 0|U| \mathrm{F} 0\rangle^{2}} \\
& \simeq 0 .
\end{aligned}
$$

The energy gap between the ground state of $S_{\text {total }}^{z}=0$ and the lowest state of $S_{\text {total }}^{z}=1$ is estimated as

$$
\langle 1 \alpha|H| 1 \alpha\rangle-\langle 0 \alpha|H| 0 \alpha\rangle \simeq \frac{1}{2}\left(E_{\mathrm{f} 1}-E_{\mathrm{f} 0}\right) .
$$

Relations between these energy gaps and the symmetry breaking of two types, namely creation of the spontaneous chirality and of the spontaneous sublattice magnetization, are discussed in Section 3 .

We give a remark for the case of the Heisenberg antiferromagnet. Since the model is isotropic, a slight modification is necessary for the approximate ground state, $\left|0 \alpha_{(\Delta=1)}\right\rangle$. We only give the form of an approximate ground state as follows:

$$
\left|0 \alpha^{\prime}\right\rangle=\frac{1}{4 \pi} \int d \Omega R(\boldsymbol{\Omega})\left|0 \alpha_{(\Delta=1)}\right\rangle / \| \frac{1}{4 \pi} \int d \Omega R(\boldsymbol{\Omega})\left|0 \alpha_{(\Delta=1)}\right\rangle \|,
$$

where $\boldsymbol{\Omega}$ is the unit vector with the spherical coordinates $(\theta, \varphi)$ and

$$
R(\boldsymbol{\Omega})=\exp \left(i \varphi \sum_{i} S_{i}^{z}\right) \exp \left(i \theta \sum_{j} S_{j}^{y}\right)
$$

We used the state $\left|0 \alpha_{(\Delta=1)}\right\rangle$ to compare our approximation with the true ground state of the Heisenberg model, for it is not easy to use $\left|0 \alpha^{\prime}\right\rangle$ in numerical calculations. 


\section{Contribution of the low-lying states to the symmetry breaking}

Several authors 12, 13, 14, 15 have discussed the mechanism of symmetry breaking in quantum Heisenberg antiferromagnets. It has been argued that, as the system size is enlarged, many low-lying states converge to the ground states and that linear combinations of these states make the symmetry breaking occur. In this section we study the roles played by the low-lying states of the two types in the symmetry breaking. Here we assume that the ground states of an infinite system have a long-range order and that the symmetry breaks down. In Section 4 we show an evidence of occurrence of the symmetry breaking.

In antiferromagnets on a triangular lattice, there are two types of symmetry breaking. Then the mechanism of symmetry breaking is complex. In the $X Y$ model, or in the $X X Z$ model for $-0.5<\Delta<1$, the $O(2)$ symmetry and the $Z_{2}$ (chiral) symmetry can break independently. In the Heisenberg model, both the spontaneous sublattice magnetization and the spontaneous chirality correspond to the breakdown of the $O(3)$ symmetry.

As shown in Section 2, the low-lying states on a finite-volume triangular lattice have the two-fold structures. The purpose of this section is to clarify the relations between the symmetry breaking of the two types and the low-lying states of the types $\alpha$ and $\beta$. Our approximation (2.2) helps us to understand the symmetry breaking of the two types independently, since the degrees of freedom of the sublattice magnetization and of the chirality are separated in it.

In an infinite system we can define four types of ground states, which are classified by existence of the spontaneous sublattice magnetization or the spontaneous chiral order. Here we show the definitions of the four types.

(1) Symmetric states (Mixed states),

$$
\langle\cdots\rangle_{1}=\lim _{N \uparrow \infty} \lim _{\beta \uparrow \infty} \frac{\operatorname{Tr}[\cdots \exp (-\beta H)]}{\operatorname{Tr}[\exp (-\beta H)]}=\omega(\cdots) .
$$

(2) States in which only the spontaneous chirality exists,

$$
\langle\cdots\rangle_{2}=\lim _{B \downarrow 0} \lim _{N \uparrow \infty} \lim _{\beta \uparrow \infty} \frac{\operatorname{Tr}\left[\cdots \exp \left(-\beta\left(H-B Q^{z}\right)\right)\right]}{\operatorname{Tr}\left[\exp \left(-\beta\left(H-B Q^{z}\right)\right)\right]}
$$

where $Q^{z}$ denotes the $z$-component of the chirality order-parameter operator

$$
\boldsymbol{Q}=\frac{2}{\sqrt{3}} \sum_{\langle i \rightarrow j\rangle}\left(\boldsymbol{S}_{i} \times \boldsymbol{S}_{j}\right)
$$

The summation is defined in the same way as in eq. (2.5).

(3) States in which only the spontaneous magnetization exists,

$$
\langle\cdots\rangle_{3}=\lim _{B \downarrow 0} \lim _{N \uparrow \infty} \lim _{\beta \uparrow \infty} \frac{\operatorname{Tr}\left[\cdots \exp \left\{-\beta\left(H-B\left(U \sum_{i} S_{i}^{x} U^{\dagger}+U^{\dagger} \sum_{i} S_{i}^{x} U\right)\right)\right\}\right]}{\operatorname{Tr}\left[\exp \left\{-\beta\left(H-B\left(U \sum_{i} S_{i}^{x} U^{\dagger}+U^{\dagger} \sum_{i} S_{i}^{x} U\right)\right)\right\}\right]} .
$$


(4) Pure states,

$$
\langle\cdots\rangle_{4}=\lim _{B \downarrow 0} \lim _{N \uparrow \infty} \lim _{\beta \uparrow \infty} \frac{\operatorname{Tr}\left[\cdots \exp \left(-\beta\left(H-B U^{\dagger} \sum_{i} S_{i}^{x} U\right)\right)\right]}{\operatorname{Tr}\left[\exp \left(-\beta\left(H-B U^{\dagger} \sum_{i} S_{i}^{x} U\right)\right)\right]}=\tilde{\omega}(\cdots) .
$$

Here the equilibrium states are defined as functionals of expectation values on an operator space. (This is familiar in studies of infinite-volume systems.) In the following, we show, using the approximate states (2.2), how each ground state is constructed of low-lying states.

(1) Symmetric states (Mixed states). By taking the thermodynamic limit of the finite-volume ground state, we get the symmetric ground state (3.1). No symmetry is broken in it.

(2) States in which only the spontaneous chirality exists. We obtain the state $\langle\cdots\rangle_{2}$ by taking the thermodynamic limit under an infinitesimal effective field which is conjugate to the chiral order parameter.

An approximation of this state is given by $|2\rangle=U|\mathrm{~F} 0\rangle$. There remains the $O(2)$ invariance in it. Both Miyashita's trial function (4) and the variational function by Huse and Elser 5 belong to this type of ground states.

In the $X Y$-like model, the state $U|\mathrm{~F} 0\rangle$ is constructed by making a linear combination of $|0 \alpha\rangle$ and $|0 \beta\rangle$,

$$
\{|0 \alpha\rangle,|0 \beta\rangle\} \longrightarrow\left\{U|\mathrm{~F} 0\rangle, \quad U^{\dagger}|\mathrm{F} 0\rangle\right\}
$$

Thus with help of our approximation we find that, if the ground state (the type $\alpha$ ) and the lowest state of the type $\beta$ become degenerate in the infinite-volume limit and if they are rearranged between themselves, the $Z_{2}$ symmetry of the chirality is broken.

In the Heisenberg model, the degree of freedom of the chirality is continuous and the ground state is isotropic, as mentioned in (2.10). Then two states are not enough to break entirely the symmetry of the chirality. Koma and Tasaki (14) proposed an approximate state which describes breakdown of continuous symmetry and they argued that the thermodynamic limit of this approximate state is a pure state, in which continuous symmetry is broken. By applying their argument to the present case, an approximate state which has fully-ordered spontaneous chirality can be constructed in the form

$$
\frac{1}{\sqrt{2 k+1}}\left\{\left|\Phi_{\mathrm{GS}}\right\rangle+\sum_{n=1}^{k}\left(\frac{\left(Q^{+}\right)^{n}\left|\Phi_{\mathrm{GS}}\right\rangle}{\|\left(Q^{+}\right)^{n}\left|\Phi_{\mathrm{GS}}\right\rangle}+\frac{\left(Q^{-}\right)^{n}\left|\Phi_{\mathrm{GS}}\right\rangle}{\|\left(Q^{-}\right)^{n}\left|\Phi_{\mathrm{GS}}\right\rangle \|}\right)\right\} .
$$

where $Q^{ \pm}=Q^{x} \pm i Q^{y}$ and $\left|\Phi_{\mathrm{GS}}\right\rangle$ denotes the ground state. The number $k$ is of $o(N)$. This state has a nonvanishing expectation value of the operator $Q^{x}$. By rotating all the spins through the angle $\pi / 2$ about the $y$ axis, we obtain an approximation for the state $\langle\cdots\rangle_{2}$. Following the arguments by Koma and Tasaki (14), the infinite-volume limit of this approximate state become $\langle\cdots\rangle_{2}$. The states $\left(Q^{ \pm}\right)^{2 m}\left|\Phi_{\mathrm{GS}}\right\rangle$ belong to the type $\alpha$ and $\left(Q^{ \pm}\right)^{2 m+1}\left|\Phi_{\mathrm{GS}}\right\rangle$ to $\beta$. Thus the state $\langle\cdots\rangle_{2}$ is constructed by a linear combination of low-lying states of the type $\alpha$ and of the type $\beta$. 
In our approximation the expectation value of the spontaneous chirality is calculated as

$$
\begin{aligned}
\left\langle 2\left|Q^{z}\right| 2\right\rangle & =\frac{E_{\mathrm{f} 0}}{J}+\frac{E_{\mathrm{f} 0}}{3 J}\langle\mathrm{~F} 0|U| \mathrm{F} 0\rangle-2 \Delta\left\langle\mathrm{F} 0\left|\sum_{\langle i, j\rangle} S_{i}^{z} S_{j}^{z}\right| \mathrm{F} 0\right\rangle \\
& \simeq \frac{E_{\mathrm{f} 0}}{J}-2 \Delta\left\langle\mathrm{F} 0\left|\sum_{\langle i, j\rangle} S_{i}^{z} S_{j}^{z}\right| \mathrm{F} 0\right\rangle
\end{aligned}
$$

where the relations $U H U^{\dagger}=\frac{1}{2} H_{\mathrm{F}}-\frac{3}{4} J Q$ and $H=-H_{\mathrm{F}}+3 J \Delta \sum_{\langle i, j\rangle} S_{i}^{z} S_{j}^{z}$ are used and $E_{\mathrm{f} 0}$ denotes the ground state energy of the ferromagnet. As $\Delta$ is increased from -0.5 , the first term in (3.8) becomes large by quantum effects. The second term in (3.8), $\left\langle\mathrm{F} 0\left|\sum_{\langle i, j\rangle} S_{i}^{z} S_{j}^{z}\right| \mathrm{F} 0\right\rangle$, behaves as follows: At $\Delta=-0.5$ this term is vanishing, since there is no correlation between up and down spins in the ground state $|\mathrm{F} 0\rangle$. As the parameter $\Delta$ increases, nearest-neighbor pairs of up and down spins are favored. Then the term becomes large. Thus at $\Delta=0$ the chirality is enhanced by the quantum effect of the first term and near $\Delta=1$ the value is reduced by contribution of the second term.

(3) States in which only the spontaneous magnetization exists. In the state $\langle\cdots\rangle_{3}$ there exists the spontaneous magnetization, though there exists no spontaneous chirality.

In the $X Y$-like model, the $O(2)$-symmetry breaking occurs in the following way. First we consider the case of the ferromagnetic $X X Z$ model (2.4). As shown in Appendix A, the finite-volume ground state of the ferromagnet is unique and it has $O(2)$-rotational invariance. It was shown in ref. 14 that the low-lying states $|\mathrm{F} n\rangle(n= \pm 1, \pm 2, \ldots, \pm o(N))$ converge to the ground state and pure infinite-volume ground states are constructed by taking linear combinations of the low-lying states. We denote one of the ferromagnetically ordered ground states as $\mid \mathrm{F}$-order $\rangle$, where $\left\langle\mathrm{F}\right.$-order $\left|S_{i}^{x}\right| \mathrm{F}$-order $\rangle \neq 0$ and $\left\langle\mathrm{F}\right.$-order $\left|S_{i}^{y}\right| \mathrm{F}$-order $\rangle=0$. In the case of the antiferromagnet, with help of the approximate low-lying states, we can understand the mechanism of the $O(2)$-symmetry breaking on the analogy of the ferromagnet. Under the effective field $B$ in (3.4), the low-lying states of the type $\alpha$ have an energy lower than those of $\beta$ have. An approximate state for $\langle\cdots\rangle_{3}$ is given by $\left(U+U^{\dagger}\right) \mid \mathrm{F}$-order $\rangle$, which is constructed by making a linear combination of $\left(U+U^{\dagger}\right)|\mathrm{F} n\rangle(n=0, \pm 1, \pm 2, \ldots)$. In the same way, by using low-lying states of the type $\beta$, another state in which only the spontaneous magnetization exists is constructed as $\left(U-U^{\dagger}\right) \mid \mathrm{F}$-order $\rangle$. Thus we find that, if the low-lying states of the same type become degenerate in the infinite-volume limit and if rearrangements occur between them, the breakdown of the $O(2)$ symmetry occurs.

For the Heisenberg model, by applying the approximation of Koma and Tasaki (14) to this model, an approximate state of $\langle\cdots\rangle_{3}$ is written as

$$
\frac{1}{\sqrt{2 k+1}}\left\{\left|\Phi_{\mathrm{GS}}\right\rangle+\sum_{n=1}^{k}\left(\frac{\left(O^{+}\right)^{n}\left|\Phi_{\mathrm{GS}}\right\rangle}{\|\left(O^{+}\right)^{n}\left|\Phi_{\mathrm{GS}}\right\rangle \|}+\frac{\left(O^{-}\right)^{n}\left|\Phi_{\mathrm{GS}}\right\rangle}{\|\left(O^{-}\right)^{n}\left|\Phi_{\mathrm{GS}}\right\rangle \|}\right)\right\}
$$

where $O^{+}=U \sum_{i} S_{i}^{+} U^{\dagger}+U^{\dagger} \sum_{i} S_{i}^{+} U$. All the low-lying states, $\left(O^{+}\right)^{m}\left|\Phi_{\mathrm{GS}}\right\rangle$ and $\left(O^{-}\right)^{m}\left|\Phi_{\mathrm{GS}}\right\rangle$, belong to the type $\alpha$. Thus We can obtain the state $\langle\cdots\rangle_{3}$ by taking a linear-combination of the low-lying states of the same type. 
(4) Pure states. The pure infinite-volume ground states have the same structure as the ground states of the classical model. The $O(2)$ symmetry and $Z_{2}$ symmetry are broken in the $X Y$ model, and the $O(3)$ symmetry of the sublattice magnetization and that of the chirality are broken in the Heisenberg model.

To construct the pure infinite-volume ground states, the symmetry breaking of two types discussed in the above should occur; A number of low-lying states of both types, $\alpha$ and $\beta$, become degenerate to the ground state and rearrangements occur between them. This is consistent with the previous arguments by Bernu et al.12), and Koma and Tasaki 14.

By discussing the symmetry breaking of two types separately, we find that the spontaneous sublattice magnetization is created by rearrangements of the low-lying states of the same type and that the spontaneous chirality is by rearrangements of pairs of low-lying states of the type $\alpha$ and of $\beta$.

It is possible that, if long-range order of one type exists in the true system, only one type of symmetry breaking occurs in the thermodynamic limit. Though the result from the renormalization group (22) indicates existence of one critical point (critical spin $S_{\mathrm{c}}$ ), our arguments suggest that symmetry breaking can occur separately and that an intermediate phase can appear in which symmetry breaking of only one type occurs.

An approximation for this state is given by

$$
|4\rangle=U \mid \mathrm{F} \text {-order }\rangle
$$

and the expectation values are related with the quantities of the ferromagnet in the forms

$$
\left\langle 4\left|U \sum_{i} S_{i}^{x} U^{\dagger}\right| 4\right\rangle=\left\langle\mathrm{F} \text {-order }\left|\sum_{i} S_{i}^{x}\right| \mathrm{F} \text {-order }\right\rangle
$$

and

$$
\left\langle 4\left|Q^{z}\right| 4\right\rangle=\left\langle 2\left|Q^{z}\right| 2\right\rangle
$$

\section{Evidence of symmetry breaking}

In this section we numerically study low-lying states of finite systems to verify the occurrence of symmetry breaking which was discussed in Section 3. We display an evidence of the symmetry breaking in the Heisenberg and $X Y$ antiferromagnets.

First we discuss the necessary conditions for symmetry breaking to occur. Bernu et al. (12) have also discussed these matters. We discuss the conditions of the creation of the spontaneous sublattice magnetization and of the spontaneous chirality separately.

When the symmetry breaking occurs in the thermodynamic limit, the low-lying states which satisfy the following two conditions should merge into the ground states? in the infinite-volume limit.

\footnotetext{
${ }^{2}$ For a definition of the ground state in quantum spin systems, see refs. 23 and 14.
} 
(1) The energy (per site) is the same as that of the ground state in the infinite-size limit.

(2) The spatial-symmetry is the same as that of the ground state of the classical model, i.e. the state has sublattice-translational invariance and $C_{3 V}$-invariance.

In the thermodynamic limit, these low-lying states form pure ground states. In general, all the pure ground states give the same physical quantities. The sublattice magnetization and the chirality per site should be the same throughout the ground states. From this consideration and the discussions in Section 3, we obtain the necessary condition of the creation of the spontaneous sublattice magnetization as follows:

(3) A number of low-lying states of the type $\alpha$ satisfy the condition (1) and they have the same macroscopic value of long-range order of the sublattice magnetization.

The necessary condition of the creation of the spontaneous chirality is as follows:

(4) Pairs of low-lying states of the type $\alpha$ and of $\beta$ satisfy the condition (1) and they have the same macroscopic value of long-range order of the chirality.

Bernu et al. (12) studied the low-lying states of the Heisenberg model on the triangular lattice. They found many low-lying states which satisfy the conditions (1) and (2), and found that these states have similar macroscopic values for the sublattice magnetization. They estimated the sublattice magnetization of the ground state in the infinite-volume limit for the Heisenberg model. Leung and Runge(9) estimated the sublattice magnetization and chirality of the ground state for the $X Y$ and Heisenberg models.

To examine these conditions explicitly, we study the energy gaps, the sublattice magnetization and the chirality of the low-lying states in the $S=1 / 2 X Y$ and Heisenberg models, and estimate the physical values of the low-lying states in the infinite-volume limit. The systems of the size $N=9,12,21$, and 27 with periodic-boundary conditions are studied, using the exact-diagonalization method. We study the low-lying states which belong to the subspaces of $S_{\text {total }}^{z}=0$ (or 0.5 ) and of $S_{\text {total }}^{z}=1$ (or 1.5), and which satisfy the condition (2). (The ground state and some low-lying excited states really belong to these subspaces.) As we have indicated in Section 2 , there exist the $C_{6}$-symmetric (type $\alpha$ ) and $C_{6}$-antisymmetric (type $\beta$ ) states. In many cases, we studied the lowest state of each subspace. There are exceptions: In the space of $S_{\text {total }}^{z}=0$ for $N=12$ and $S_{\text {total }}^{z}=1.5$ for $N=21$ of the Heisenberg model, we chose the first-excited state of the type $\beta$, since the first-excited state of the type $\alpha$ and the lowest state of the type $\beta$ are degenerate and form a paired doublet, and the ground state (the type $\alpha$ ) and the first excited state of the type $\beta$ are in pairs.

The lowest state in the $S_{\text {total }}^{z}=0$ and $C_{6}$-symmetric (type $\alpha$ ) subspace is the ground state, which has been already calculated up to $N=36$ in refs. 12 and 9 . We used the data for $N=36$ in ref. 9 .

First we discuss the energy gap. Here the energy gap is defined as the difference between the total energies of two states. The energy gap between the lowest state of the type $\alpha$ and that of 
$\beta$ is shown in Fig. 2. In many cases the states of the type $\alpha$ and $\beta$ are degenerate. The energy gap between the ground state (the type $\alpha$ ) and the lowest state (the type $\alpha$ ) of the $S_{\text {total }}^{z}=1$ subspace is plotted in Fig. 3. It decreases proportionally to $N^{-1}$. As we have discussed in Section 3, the former energy gap is relevant to the symmetry breaking of the chirality and the latter is to the breakdown of the rotational symmetry which creates the spontaneous sublattice magnetization. The latter energy gap corresponds to the singlet-triplet gap in the Heisenberg model, which was reported in ref. 9.

It was proved that, if there is a long-range order, the energy gap decreases in the $N^{-1}$ form or faster than it. (13, 14) Thus the above calculated results indicate an evidence for the existence of long-range orders in the $X Y$ and Heisenberg antiferromagnets.

Next we consider the sublattice magnetization which is observed with the operator

$$
M=\sum_{i \in A} S_{i}^{x}+\sum_{i \in B}\left(-\frac{1}{2} S_{i}^{x}+\frac{\sqrt{3}}{2} S_{i}^{y}\right)+\sum_{i \in C}\left(-\frac{1}{2} S_{i}^{x}-\frac{\sqrt{3}}{2} S_{i}^{y}\right) .
$$

We calculated the long-range order of the sublattice magnetization $\left\langle M^{2}\right\rangle / N^{2}$. The values of the spontaneous sublattice magnetization $m$ are estimated, using the relation $m=\lim _{N \uparrow \infty} \sqrt{6\left\langle M^{2}\right\rangle} / N$ for the Heisenberg model and $m=\lim _{N \uparrow \infty} 2 \sqrt{\left\langle M^{2}\right\rangle} / N$ for the $X Y$ model. We show a derivation of these relations in Appendix B. We fit the data of the sublattice magnetization $m$ to the $N^{-1 / 2}$ form. This finite-size correction was derived from the spin-wave theory 24 and using the effective Hamiltonian for a large spin(16). (We also fit the long-range correlation $\left\langle M^{2}\right\rangle / N^{2}$ to the $N^{-1 / 2}$ form, using the data for $N=3,9,12,21$, and 27 , and found that the correction term of $O\left(N^{-1}\right)$ in the fitting of $\left\langle M^{2}\right\rangle / N^{2}$ is larger than that in the fitting of $m$. Therefore we adopted the fitting of $m$.) The results for the $X Y$ model are shown in Fig. 4. We extrapolated in the $N^{-1 / 2}$ form, using the data for $N=9,12,21$, and 27. For the ground state we also used the data for $N=36$ which was reported in ref. 9. The values in the infinite-volume limit are shown in Table 2. They coincide with each other. Thus the condition (3) is satisfied in the XY model. The data for the Heisenberg model are shown in Fig. 5. The values in the infinite-size limit are shown in Table 3. They are almost equal. Thus the condition (3) is also satisfied in the Heisenberg model. All the values are nonvanishing and close to the result by the spin-wave theory. However, as it is reported in ref. 9, if we fit the data $\left\langle M^{2}\right\rangle / N^{2}$ to $N^{-1 / 2}$, we obtain the estimate $m \simeq 0$. The values seriously depend on the fitting form. Thus it is still hard to conclude the existence of the long-range order in the Heisenberg model.

Finally we consider the chiral order, which is observed with the operator

$$
\boldsymbol{Q}=\frac{2}{\sqrt{3}} \sum_{<i \rightarrow j>} \boldsymbol{S}_{i} \times \boldsymbol{S}_{j}
$$

where the symbol $i \rightarrow j$ goes from the sublattice $\mathrm{A}$ to $\mathrm{B}, \mathrm{B}$ to $\mathrm{C}$, and $\mathrm{C}$ to $\mathrm{A}$. We calculated the long-range order of the chirality $\left\langle\left(Q^{z}\right)^{2}\right\rangle / N^{2}$. The values of the spontaneous chirality $q$ are estimated using the relations $q=\lim _{N \uparrow \infty} \sqrt{3\left\langle\left(Q^{z}\right)^{2}\right\rangle} / N$ for the Heisenberg model and $q=$ $\lim _{N \uparrow \infty} \sqrt{\left\langle\left(Q^{z}\right)^{2}\right\rangle} / N$ for the $X Y$ model. We show the results for the $X Y$ model in Fig. 6 and for 
Table 2: Estimates of the sublattice magnetization $m$ and the chirality $q$ for the $S=1 / 2-X Y$ antiferromagnet on the triangular lattice.

\begin{tabular}{lcc}
\hline \hline & $m$ & $q$ \\
\hline$S_{\text {total }}^{z}=0$, Type $\alpha$ & 0.41 & 0.76 \\
$S_{\text {total }}^{z}=0$, Type $\beta$ & 0.39 & 0.76 \\
$S_{\text {total }}^{z}=1$, Type $\alpha$ & 0.42 & 0.77 \\
$S_{\text {total }}^{z}=1$, Type $\beta$ & 0.41 & 0.78 \\
\hline Spin-wave expansion & $0.437($ ( $)$ & $0.798($ 目 \\
\hline \hline
\end{tabular}

the Heisenberg model in Fig. 7. For the $X Y$ model we extrapolated the expectation values of the chirality in the $N^{-3 / 2}$ form, which we derive from the finite-size correction of the spin-wave theory 8 . On the other hand, in the Heisenberg model the finite-size correction behaves in the $N^{-1 / 2}$ form, as discussed by Azaria et al. (16) This difference of the correction terms comes from the fact that in the Heisenberg model the chirality is sensitive to spin-wave fluctuations of long wave-length, while in the $X Y$ model the chiral order is stable against them. The extrapolated values are shown in Tables 2 and 3 . In the $X Y$ model the values are consistent with each other and nonvanishing, which suggests the existence of the chiral order. Thus the condition (4) is satisfied. In the Heisenberg model the values do not agree with each other. It is necessary to calculate larger systems to conclude that these low-lying states have the same chirality. We can not fit the data to a form including higher-order terms, since the size of the data is too small. Thus we can not still conclude the existence of the long-range order of the chirality in the Heisenberg model.

To test the mechanism of symmetry-breaking of the chirality which we have discussed in

Table 3: Estimates of the sublattice magnetization $m$ and the chirality $q$ for the $S=1 / 2$ Heisenberg antiferromagnet on the triangular lattice.

\begin{tabular}{lcc}
\hline \hline & $m$ & $q$ \\
\hline$S_{\text {total }}^{z}=0$, Type $\alpha$ & 0.24 & 0.21 \\
$S_{\text {total }}^{z}=0$, Type $\beta$ & 0.22 & 0.16 \\
$S_{\text {total }}^{z}=1$, Type $\alpha$ & 0.24 & 0.54 \\
$S_{\text {total }}^{z}=1$, Type $\beta$ & 0.38 & 0.73 \\
\hline Spin-wave expansion & 0.250 (日) & 0.405 ( $)$ \\
\hline \hline
\end{tabular}


Section 3, we construct the following state

$$
|\phi\rangle=\frac{1}{\sqrt{2}}(|\alpha\rangle+i|\beta\rangle),
$$

where $|\alpha\rangle(|\beta\rangle)$ denotes the lowest state of the type $\alpha(\beta)$ of each $S_{\text {total }}^{z}$ subspace. We observe the spontaneous chirality of this state. The expectation value of the chirality is calculated through the relation

$$
\left\langle\phi\left|Q^{z}\right| \phi\right\rangle=i\left\langle\alpha\left|Q^{z}\right| \beta\right\rangle
$$

The estimates in the $X Y$ model are shown in Fig. 8. They are almost equal to the values which are shown in Fig. 6. This coincidence suggests correctness of the mechanism of the chiral symmetry breaking which was discussed in Section 3. In the Heisenberg model we multiply the factor $\sqrt{3}$ to the calculated values, since the symmetry is not fully broken in the state (4.3); the state (3.7) should be properly used instead of (4.3). The estimates are shown in Fig. 9. Thus we can break the chiral symmetry by making a linear combination of low-lying states of the type $\alpha$ and of the type $\beta$.

From the above results we summarize that the necessary conditions (3) and (4) are satisfied in the $X Y$ model. In the Heisenberg model the necessary conditions are almost satisfied, though there still remains ambiguity in the point that the low-lying states have the same properties. In any case, in the above results there is no evidence which contradicts the occurrence of symmetry breaking.

\section{Summary}

To summarize, we studied the low-lying states of quantum antiferromagnets on a triangular lattice to clarify what kind of roles they play in the symmetry breaking and to show an evidence of occurrence of the symmetry breaking.

We gave approximate forms of low-lying states, which have two-fold structures. It was found that these approximate states resemble the true states in various properties. We classified the low-lying states by the eigenvalues of $C_{6}$, namely $C_{6}$-symmetric and $C_{6}$-antisymmetric. States of both types exist in pairs in the true low-lying spectrum. We discussed how rearrangements of the low-lying states of two types bring on the symmetry breaking. The spontaneous chirality

is created by taking a linear combination of pairs of low-lying state of the type $\alpha$ and of $\beta$. The spontaneous sublattice magnetization is obtained by making a linear combination of low-lying states of the same type.

To display an evidence of the occurrence of symmetry breaking, we studied low-lying states of finite systems using the exact-diagonalization method. We studied whether the necessary conditions of the symmetry breaking are satisfied or not. We found that the conditions are satisfied in the $X Y$ antiferromagnet. The estimate of the sublattice magnetization is $m=0.41$ and of chirality is $q=0.77$. These values are less than the spin-wave results by $7 \%$ and $4 \%$ 
respectively. In the Heisenberg antiferromagnet the conditions are almost satisfied, although the estimates of the sublattice magnetization and the chirality do not converge well for lack of the system-size. The sublattice magnetization is estimated as $m=0.22 \sim 0.38$ and the chirality is $q=0.16 \sim 0.73$, where the results from the spin-wave expansion are $m=0.25$ and $q=0.45$. Since the extrapolated results seriously depend on fitting forms, it is still hard in the present study to conclude definitely the existence of the long-range order in the Heisenberg model.

\section{Appendix A. Low-lying states of the ferromagnetic XXZ model}

We show the uniqueness of the ground state and give spatial quantum numbers of the low-lying states of the spin $S X X Z$ ferromagnet on finite systems. We discuss the model on the finitevolume triangular lattice $\Lambda$ with a periodic boundary condition. For simplicity, we restrict the number of sites to an even integer. The Hamiltonian is given by

$$
H_{F}=-J \sum_{\langle i, j\rangle \in \Lambda}\left(S_{i}^{x} S_{j}^{x}+S_{i}^{y} S_{j}^{y}+\eta S_{i}^{z} S_{j}^{z}\right)
$$

where $J>0$ and $\eta<1$. The summation runs over all the nearest-neighbor sites.

The Hamiltonian (A.1) has nonpositive off-diagonal elements. The basis which have the

same $S_{\text {total }}^{z}$ are connected by the elements of the Hamiltonian. From the Perron-Frobenius theorem, the lowest eigenstate in each $S_{\text {total }}^{z}$ subspace is unique and it has nonnegative coefficients.

Here we show that the ground state exists uniquely in the $S_{\text {total }}^{z}=0$ subspace. We consider the following Hamiltonian

$$
H_{F}^{\prime}=-\sum_{i, j \in \Lambda} J_{i j}\left(S_{i}^{x} S_{j}^{x}+S_{i}^{y} S_{j}^{y}+\eta S_{i}^{z} S_{j}^{z}\right)
$$

where $J_{i j} \geq 0$ for all $i$ and $j$. Affleck and Lieb25 showed that, in the antiferromagnetic $X X Z$ chain, the ground state exists uniquely in the $S_{\text {total }}^{z}=0$ subspace. As they did, we transform the Hamiltonian (A.2) using the unitary operator

$$
W=\exp \left(i \frac{\pi}{2} \sum_{i} S_{i}^{x}\right)
$$

into the form

$$
W^{\dagger} H_{F}^{\prime} W=-J \sum_{i, j \in \Lambda}\left\{\frac{1}{4}(1+\eta)\left(S_{i}^{+} S_{j}^{-}+S_{i}^{-} S_{j}^{+}\right)+\frac{1}{4}(1-\eta)\left(S_{i}^{+} S_{j}^{+}+S_{i}^{-} S_{j}^{-}\right)+S_{i}^{z} S_{j}^{z}\right\} .
$$

In the region $-1<\eta<1$, this transformed Hamiltonian has nonpositive off-diagonal elements. It has two connected blocks, i.e. all basis in the subspaces with even $S_{\text {total }}^{z}$ are connected by 
the elements of the Hamiltonian and those with odd $S_{\text {total }}^{z}$ are connected. From the PerronFrobenius theorem, the lowest eigenstate in each connected subspace is unique. Thus we find that the number of the ground states is at most two in the original model (A.2). The lowest states in the $S_{\text {total }}^{z}=n$ and $S_{\text {total }}^{z}=-n$ subspaces are degenerate. When level crossing of the ground state occurs, more than two ground states must exist, which contradicts the above result. Thus in the whole region of various parameters $\left\{J_{i j}\right\}$, the ground state should exist in the same $S_{\text {total }}^{z}$ subspace.

For the region $\eta<-1$, as Affleck and Lieb did, the above results can be shown using the unitary operator

$$
W=\exp \left(i \frac{\pi}{2} \sum_{i} S_{i}^{y}\right)
$$

To obtain the eigenvalue $S_{\text {total }}^{z}$ of the ground state, we consider the case $J_{i j}=J$ for all $i$ and $j$. The Hamiltonian can be written in the form

$$
H_{F}^{\prime}=-J\left\{(\boldsymbol{S})^{2}-(1-\eta)\left(S^{z}\right)^{2}\right\}
$$

where $\boldsymbol{S}=\left(S^{x}, S^{y}, S^{z}\right)$ and $S^{\alpha}=\sum_{i \in \Lambda} S_{i}^{\alpha}(\alpha=x, y, z)$. The ground state of this model is

unique and it has the eigenvalues $S_{\text {total }}=S N$ and $S_{\text {total }}^{z}=0$. 26) From the above results, we find that the ground state of the original model (A.1) in the regions $\eta<-1$ and $-1<\eta<1$ exists uniquely in the $S_{\text {total }}^{z}=0$ subspace.

This argument can be extended to the ferromagnetic $X X Z$ model on any lattice. The ferromagnet on a bipartite lattice is equivalent to the $X X Z$ antiferromagnet with the $z$-component anisotropy $-\eta$. In this case the above results are consistent with what were proved by Lieb and Mattis (27), and Affleck and Lieb 25).

Lastly, we give the spatial quantum numbers of the lowest state in each $S_{\text {total }}^{z}$ subspace. The Hamiltonian (A.1) is invariant under any translation, rotation, and reflection. As we have shown in the above, the lowest state is nondegenerate and it has nonnegative coefficients. From this fact, we find that the lowest state has the eigenvalue 1 for any translation, rotation, and reflection, i.e. it is translationally invariant and $C_{6 V}$-invariant.

\section{Appendix B. Relations between long-range order and symmetry breaking}

We discuss the relation between spontaneous symmetry breaking $m$ and the long-range order parameter $\sigma$. It has been discussed (28, 17) that the relation is given by $m=\sqrt{3} \sigma$ for the Heisenberg antiferromagnet and by $m=\sqrt{2} \sigma$ for the $X Y$ antiferromagnet on bipartite lattices. But these relations are not valid in the antiferromagnets on the triangular lattice. Here we show that, in the antiferromagnets on the triangular lattice, the factor becomes $\sqrt{2}$ times as large as the above. 
Our arguments are based on the assumption that the symmetric infinite-volume ground state, (3.1), is decomposed into the pure ground states. Koma and Tasaki 17 used this decomposition to explain the factor $\sqrt{3}$ for the Heisenberg antiferromagnets on bipartite systems.

The spontaneous sublattice magnetization $m$ is defined as

$$
m \equiv \lim _{B \downarrow 0} \lim _{N \uparrow \infty} \lim _{\beta \uparrow \infty} \frac{1}{N} \frac{\operatorname{Tr}[M \exp \{-\beta(H-B M)\}]}{\operatorname{Tr}[\exp \{-\beta(H-B M)\}]} .
$$

Using the sublattice-translational invariance and equivalence of the sublattice magnetization, we have

$$
m=\tilde{\omega}\left(S_{0}^{x}\right),
$$

where the state $\tilde{\omega}(\cdots)$ is defined by $(3.5)$. The long-range order parameter of the sublattice magnetization $\sigma_{m}$ is defined by

$$
\sigma_{m}^{2} \equiv \lim _{N \uparrow \infty} \lim _{\beta \uparrow \infty} \frac{1}{N^{2}} \frac{\operatorname{Tr}\left[M^{2} \exp (-\beta H)\right]}{\operatorname{Tr}[\exp (-\beta H)]} .
$$

Using the rotational and translational invariance, we obtain

$$
\begin{aligned}
& \sigma_{m}^{2}=\lim _{|r| \uparrow \infty} \frac{1}{3} \quad\left\{\omega\left(S_{0}^{x} S_{r}^{x}\right)+\omega\left(-\frac{1}{2} S_{0}^{x} S_{r+\boldsymbol{e}_{1}}^{x}+\frac{\sqrt{3}}{2} S_{0}^{x} S_{r+\boldsymbol{e}_{1}}^{y}\right)\right. \\
& \left.+\omega\left(-\frac{1}{2} S_{0}^{x} S_{r+\boldsymbol{e}_{2}}^{x}-\frac{\sqrt{3}}{2} S_{0}^{x} S_{r+\boldsymbol{e}_{2}}^{y}\right)\right\},
\end{aligned}
$$

where the state $\omega$ is defined by (3.1). The sites 0 and $r$ belong to the sublattice A and $\boldsymbol{e}_{\mathbf{1}}\left(\boldsymbol{e}_{\mathbf{2}}\right)$ denotes the unit lattice vector, which belongs to the sublattice $\mathrm{B}(\mathrm{C})$.

We also define the spontaneous chirality $q$ as

$$
q \equiv \lim _{B \downarrow 0} \lim _{N \uparrow \infty} \lim _{\beta \uparrow \infty} \frac{1}{N} \frac{\operatorname{Tr}\left[Q^{z} \exp \{-\beta(H-B M)\}\right]}{\operatorname{Tr}[\exp \{-\beta(H-B M)\}]}=\tilde{\omega}\left(Q^{z}(0)\right)
$$

and the long-range order parameter of the chirality $\sigma_{q}$ as

$$
{\sigma_{q}}^{2} \equiv \lim _{N \uparrow \infty} \lim _{\beta \uparrow \infty} \frac{1}{N^{2}} \frac{\operatorname{Tr}\left[\left(Q^{z}\right)^{2} \exp (-\beta H)\right]}{\operatorname{Tr}[\exp (-\beta H)]}=\lim _{|r| \uparrow \infty} \omega\left(Q^{z}(0) Q^{z}(r)\right),
$$

where the operator $Q^{z}(r)$ is the z-component of the chiral order-parameter operator on the unit triangular cell,

$$
\boldsymbol{Q}(r)=\frac{2}{\sqrt{3}}\left(\boldsymbol{S}_{r} \times \boldsymbol{S}_{r+\boldsymbol{e}_{1}}+\boldsymbol{S}_{r+\boldsymbol{e}_{1}} \times \boldsymbol{S}_{r+\boldsymbol{e}_{2}}+\boldsymbol{S}_{r+\boldsymbol{e}_{2}} \times \boldsymbol{S}_{r}\right) .
$$

From now we derive the relations between $m$ and $\sigma_{m}$, and between $q$ and $\sigma_{q}$. Our results are as follows: For the $X Y$ model

$$
m=2 \sigma_{m} \quad \text { and } \quad q=\sigma_{q},
$$

and for the Heisenberg model

$$
m=\sqrt{6} \sigma_{m} \quad \text { and } \quad q=\sqrt{3} \sigma_{q} .
$$


The relation $m=\sqrt{6} \sigma_{m}$ for the Heisenberg model has been already used by Bernu et al. (12).

To derive these relations, we use the following standard arguments. It is widely believed that the mixed state $\omega$ can be naturally decomposed into pure equilibrium states, 23)

$$
\omega(\cdots)=\int d \alpha \omega_{\alpha}(\cdots)
$$

where $\left\{\omega_{\alpha}\right\}$ denote the pure states and the parameter $\alpha$ describes the properties of them. The state $\tilde{\omega}$, which is defined in eq. (3.5), is one of $\omega_{\alpha}$. The pure states have the cluster property, (23)

$$
\omega_{\alpha}\left(A_{0} B_{r}\right) \underset{|r| \uparrow \infty}{\longrightarrow} \omega_{\alpha}\left(A_{0}\right) \omega_{\alpha}\left(B_{r}\right)
$$

for any local operators $A$ and $B$.

\section{B.1 XY-like model}

In the $X X Z$ model with $-0.5<\Delta<1$, it is expected that the decomposition (B.10) is of the form

$$
\omega(\cdots)=\frac{1}{4 \pi} \int_{0}^{2 \pi} d \theta\left\{\omega_{\theta,+}(\cdots)+\omega_{\theta,-}(\cdots)\right\}
$$

where $\omega_{\theta,+}\left(\omega_{\theta,-}\right)$ denote the states which have positive(negative) chirality and in which vectors of spins on the sublattice A form the angle $\theta$ with the $x$-axis. The state $\tilde{\omega}$ corresponds to $\omega_{\theta=0,+}$. The pure states have following expectation values of the sublattice magnetization

$$
\begin{aligned}
\omega_{\theta,+}\left(S_{0}^{x}\right) & =m \cos \theta, & \omega_{\theta,-}\left(S_{0}^{x}\right)=m \cos \theta, \\
\omega_{\theta,+}\left(-\frac{1}{2} S_{\boldsymbol{e}_{\mathbf{1}}}^{x}+\frac{\sqrt{3}}{2} S_{\boldsymbol{e}_{\mathbf{1}}}^{y}\right) & =m \cos \theta, & \omega_{\theta,-}\left(-\frac{1}{2} S_{\boldsymbol{e}_{\mathbf{1}}}^{x}+\frac{\sqrt{3}}{2} S_{\boldsymbol{e}_{\mathbf{1}}}^{y}\right)=m \cos \left(\theta-\frac{2 \pi}{3}\right), \\
\omega_{\theta,+}\left(-\frac{1}{2} S_{\boldsymbol{e}_{\mathbf{2}}}^{x}-\frac{\sqrt{3}}{2} S_{\boldsymbol{e}_{\mathbf{2}}}^{y}\right) & =m \cos \theta, & \omega_{\theta,-}\left(-\frac{1}{2} S_{\boldsymbol{e}_{\mathbf{2}}}^{x}-\frac{\sqrt{3}}{2} S_{\boldsymbol{e}_{\mathbf{2}}}^{y}\right)=m \cos \left(\theta+\frac{2 \pi}{3}\right),
\end{aligned}
$$

and the chirality

$$
\omega_{\theta, \pm}\left(Q^{z}(0)\right)= \pm q
$$

Using the decomposition $(\overline{B .12})$ and the property $(\underline{B .11})$, the long range order parameter of the sublattice magnetization, (B.4), is transformed as

$$
\begin{aligned}
\sigma_{m}^{2}= & \lim _{|r| \uparrow \infty} \frac{1}{12 \pi} \int_{0}^{2 \pi} d \theta\left\{\omega_{\theta,+}\left(S_{0}^{x} S_{r}^{x}\right)+\omega_{\theta,+}\left(-\frac{1}{2} S_{0}^{x} S_{r+\boldsymbol{e}_{1}}^{x}+\frac{\sqrt{3}}{2} S_{0}^{x} S_{r+\boldsymbol{e}_{1}}^{y}\right)\right. \\
& +\omega_{\theta,+}\left(-\frac{1}{2} S_{0}^{x} S_{r+\boldsymbol{e}_{2}}^{x}-\frac{\sqrt{3}}{2} S_{0}^{x} S_{r+\boldsymbol{e}_{2}}^{y}\right)+\omega_{\theta,-}\left(S_{0}^{x} S_{r}^{x}\right) \\
& \left.+\omega_{\theta,-}\left(-\frac{1}{2} S_{0}^{x} S_{r+\boldsymbol{e}_{1}}^{x}+\frac{\sqrt{3}}{2} S_{0}^{x} S_{r+\boldsymbol{e}_{1}}^{y}\right)+\omega_{\theta,-}\left(-\frac{1}{2} S_{0}^{x} S_{r+\boldsymbol{e}_{2}}^{x}-\frac{\sqrt{3}}{2} S_{0}^{x} S_{r+\boldsymbol{e}_{2}}^{y}\right)\right\} \\
= & \frac{1}{12 \pi} \int_{0}^{2 \pi} d \theta \\
& {\left[\omega_{\theta,+}\left(S_{0}^{x}\right)\left\{\omega_{\theta,+}\left(S_{0}^{x}\right)+\omega_{\theta,+}\left(-\frac{1}{2} S_{\boldsymbol{e}_{1}}^{x}+\frac{\sqrt{3}}{2} S_{\boldsymbol{e}_{1}}^{y}\right)+\omega_{\theta,+}\left(-\frac{1}{2} S_{\boldsymbol{e}_{2}}^{x}-\frac{\sqrt{3}}{2} S_{\boldsymbol{e}_{2}}^{y}\right)\right\}\right.}
\end{aligned}
$$




$$
\begin{aligned}
& \left.+\omega_{\theta,-}\left(S_{0}^{x}\right)\left\{\omega_{\theta,-}\left(S_{0}^{x}\right)+\omega_{\theta,-}\left(-\frac{1}{2} S_{\boldsymbol{e}_{1}}^{x}+\frac{\sqrt{3}}{2} S_{\boldsymbol{e}_{1}}^{y}\right)+\omega_{\theta,-}\left(-\frac{1}{2} S_{\boldsymbol{e}_{2}}^{x}-\frac{\sqrt{3}}{2} S_{\boldsymbol{e}_{2}}^{y}\right)\right\}\right] \\
= & \frac{m^{2}}{4 \pi} \int_{0}^{2 \pi} d \theta \cos ^{2} \theta=\frac{m^{2}}{4} .
\end{aligned}
$$

Thus we obtain the relation $m=2 \sigma_{m}$.

In the same way, the long-range order parameter of the chirality, $(\overline{\mathrm{B} .6})$, is estimated as

$$
\begin{aligned}
\sigma_{q}^{2} & =\lim _{|r| \uparrow \infty} \frac{1}{4 \pi} \int_{0}^{2 \pi} d \theta\left\{\omega_{\theta,+}\left(Q^{z}(0) Q^{z}(r)\right)+\omega_{\theta,-}\left(Q^{z}(0) Q^{z}(r)\right)\right\} \\
& =\frac{1}{4 \pi} \int_{0}^{2 \pi} d \theta\left\{\omega_{\theta,+}\left(Q^{z}(0)\right)^{2}+\omega_{\theta,-}\left(Q^{z}(0)\right)^{2}\right\}=q^{2} .
\end{aligned}
$$

Thus we get the relation $q=\sigma_{q}$.

\section{B.2 Heisenberg model}

For the Heisenberg model it is expected that the mixed state $\omega(\cdots)$ is decomposed as

$$
\omega(\cdots)=\frac{1}{8 \pi^{2}} \int d \Omega \int_{0}^{2 \pi} d \phi \omega_{\Omega, \phi}(\cdots),
$$

where the vector $\Omega$ is perpendicular to the plane to which all spins are parallel, and the angle $\phi$ denotes rotation with respect to the vector $\Omega$. The direction of the vector $\Omega$ and that of the chirality are the same, which are described with the spherical coordinates $(\theta, \varphi)$. The state $\tilde{\omega}$ corresponds to the state $\omega_{\substack{\varphi=0 \\ \phi=0}}$. The pure states have following expectation values of the sublattice magnetization

$$
\begin{aligned}
& \omega_{\boldsymbol{\Omega}, \phi}\left(S_{0}^{x}\right)=m(\cos \varphi \cos \theta \cos \phi-\sin \varphi \sin \phi), \\
& \omega_{\boldsymbol{\Omega}, \phi}\left(-\frac{1}{2} S_{\boldsymbol{e}_{\mathbf{1}}}^{x}+\frac{\sqrt{3}}{2} S_{\boldsymbol{e}_{\mathbf{1}}}^{y}\right) \\
& =m\left\{\cos \left(\varphi-\frac{2 \pi}{3}\right) \cos \theta \cos \left(\phi+\frac{2 \pi}{3}\right)-\sin \left(\varphi-\frac{2 \pi}{3}\right) \sin \left(\phi+\frac{2 \pi}{3}\right)\right\}, \\
& \omega_{\boldsymbol{\Omega}, \phi}\left(-\frac{1}{2} S_{\boldsymbol{e}_{\mathbf{2}}}^{x}-\frac{\sqrt{3}}{2} S_{\boldsymbol{e}_{\mathbf{2}}}^{y}\right) \\
& =m\left\{\cos \left(\varphi+\frac{2 \pi}{3}\right) \cos \theta \cos \left(\phi-\frac{2 \pi}{3}\right)-\sin \left(\varphi+\frac{2 \pi}{3}\right) \sin \left(\phi-\frac{2 \pi}{3}\right)\right\},
\end{aligned}
$$

and the chirality

$$
\omega_{\boldsymbol{\Omega}, \phi}(\boldsymbol{Q}(0))=q \boldsymbol{\Omega} .
$$

Using the decomposition (B.17) and the property (B.11), the long-range order parameter of the sublattice magnetization is transformed as

$$
\begin{aligned}
\sigma_{m}^{2}= & \frac{1}{24 \pi^{2}} \int d \Omega \int_{0}^{2 \pi} d \phi \omega_{\boldsymbol{\Omega}, \phi}\left(S_{0}^{x}\right) \\
& \times\left\{\omega_{\boldsymbol{\Omega}, \phi}\left(S_{0}^{x}\right)+\omega_{\boldsymbol{\Omega}, \phi}\left(-\frac{1}{2} S_{\boldsymbol{e}_{1}}^{x}+\frac{\sqrt{3}}{2} S_{\boldsymbol{e}_{1}}^{y}\right)+\omega_{\boldsymbol{\Omega}, \phi}\left(-\frac{1}{2} S_{\boldsymbol{e}_{2}}^{x}-\frac{\sqrt{3}}{2} S_{\boldsymbol{e}_{2}}^{y}\right)\right\} \\
= & \frac{m^{2}}{6} .
\end{aligned}
$$


Thus we obtain the relation $m=\sqrt{6} \sigma_{m}$.

Using the decomposition $(\overline{B .17})$ and the property (B.11), the long-range order parameter of the chirality is transformed as

$$
\begin{aligned}
\sigma_{q}^{2} & =\frac{1}{3} \lim _{|r| \uparrow \infty} \omega(\boldsymbol{Q}(0) \boldsymbol{Q}(r)) \\
& =\frac{q^{2}}{24 \pi^{2}} \int d \Omega \int_{0}^{2 \pi} d \phi \boldsymbol{\Omega}^{2}=\frac{q^{2}}{3}
\end{aligned}
$$

where the isotropy of the state is used. Thus we get the relation $q=\sqrt{3} \sigma_{q}$.

\section{Acknowledgments}

The author would like to thank Prof. M. Suzuki and Dr. N. Hatano for helpful discussions and critically reading this manuscript. The author is also grateful to Dr. T. Koma, Prof. H. Nishimori and Dr. Y. Nonomura for fruitful comments. Subroutines of TITPACK Ver.2 by Prof. H. Nishimori were partly used. The numerical calculations have been performed with the HITAC S820/80 of the Computer Center, University of Tokyo and the VAX 6440 of the Meson Science Laboratory, Faculty of Science, University of Tokyo. This study is partially financed by Grantin-Aid for Scientific Research on Priority Areas "Computational Physics as a New Frontier in Condensed Matter Research", from the Ministry of Education, Science and Culture, Japan.

\section{References}

[1] P. W. Anderson, Mat. Res. Bull. 8, 153 (1973).

[2] P. Fazekas and P. W. Anderson, Phil. Mag. 30, 423 (1974).

[3] V. Kalmeyer and R. B. Laughlin, Phys. Rev. Lett. 59, 2095 (1987); Phys. Rev. B 39, 11879 (1989).

[4] S. Miyashita, J. Phys. Soc. Jpn. 53, 44 (1984).

[5] D. A. Huse and V. Elser, Phys. Rev. Lett. 60, 2531 (1988).

[6] T. Oguchi, Proc. Int. Meet. Transition to "New Type of Ordered Phase", Kyoto, 1982, J. Phys. Soc. Jpn. 52 Suppl., P. 183 (1983).

[7] S. J. Miyake, J. Phys. Soc. Jpn. 61, 983 (1992).

[8] T. Momoi and M. Suzuki, J. Phys. Soc. Jpn. 61, 3732 (1992).

[9] P. W. Leung and K. J. Runge, Phys. Rev. B 47, 5861 (1993). 
[10] R. R. P. Singh and D. A. Huse, Phys. Rev. Lett. 68, 1766 (1992).

[11] H. Nishimori and H. Nakanishi, J. Phys. Soc. Jpn. 57, 626 (1988); ibid. 58, 3433 (1989).

[12] B. Bernu, C. Lhuillier, and L. Pierre, Phys. Rev. Lett. 69, 2590 (1992).

[13] P. Horsch and W. von der Linden, Z. Phys. B 72, 181 (1988).

[14] T. Koma and H. Tasaki, Obscured symmetry breaking and low-lying states in quantum many-body systems, preprint.

[15] S. Tang and J. E. Hirsch, Phys. Rev. B 39, 4548 (1989).

[16] P. Azaria, B. Delamotte, and D. Mouhanna, Phys. Rev. Lett. 70, 2483 (1993).

[17] T. Koma and H. Tasaki, Phys. Rev. Lett. 70, 93 (1992); Symmetry breaking in Heisenberg antiferromagnets, preprint.

[18] S. Miyashita, Prog. Theor. Phys. Suppl. 87, 112 (1986).

[19] H. Nishimori and S. J. Miyake, Prog. Theor. Phys. 73, 18 (1985).

[20] Z. Weihong, J. Oittmaa, and C. J. Hamer, Phys. Rev. B 44, 11869 (1991).

[21] D. D. Betts and S. Miyashita, Can. J. Phys. 68, 1410 (1990).

[22] P. Azaria, B. Delamotte, and D. Mouhanna, Phys. Rev. Lett. 68, 1762 (1992).

[23] O. Bratteli, D. W. Robinson, Operator algebras and quantum statistical mechanics I, II, (Springer, 1979).

[24] D. A. Huse, Phys. Rev. B 37, 2380 (1988).

[25] I. Affleck and E. H. Lieb, Lett. Math. Phys. 12, 57 (1986).

[26] M. Suzuki and S. Miyashita, Can. J. Phys. 56, 902 (1978).

[27] E. H. Lieb and D. Mattis, J. Math. Phys. 3, 749 (1962).

[28] S. Miyashita, in Proceedings of the Workshop on Quantum Simulations of Condensed Matter Phenomena, eds. J .D Doll and J. E. Gubernatis, p. 228 (World Scientific, 1989). 


\section{Figure Captions}

Figure 1: Two-fold structures in the spectrum of low-lying states.

Figure 2: Size dependence of the energy gap between the lowest state (the type $\alpha$ ) and the lowest excited state (the type $\beta$ ) in the same $S_{\text {total }}^{z}$-subspace for the Heisenberg model.

Figure 3: Size dependence of the energy gap between the ground state (the type $\alpha$ ) and the lowest state (the type $\alpha$ ) in $S_{\text {total }}^{z}=1$.

Figure 4: Size dependence of the sublattice magnetization of the lowest state in each subspace of the $X Y$ model, which is estimated through $2 \sqrt{\left\langle M^{2}\right\rangle} / N$. The data for $N=36$ is also listed(12). SW denotes the result from the spin-wave expansion $\left(\mathrm{B}^{\mathrm{B}}\right.$.

Figure 5: Size dependence of the sublattice magnetization of the lowest state in each subspace of the Heisenberg model, which is estimated through $\sqrt{6\left\langle M^{2}\right\rangle} / N$. The data for $N=36$ is also listed (12). SW denotes the result from the spin-wave expansion H) $^{2}$.

Figure 6: Size dependence of the chirality of the lowest state in each subspace of the $X Y$ model which is estimated through $\sqrt{\left\langle\left(Q^{z}\right)^{2}\right\rangle} / N$. The data for $N=36$ is also listed 122. SW denotes the result from the spin-wave expansion li $^{\circ}$.

Figure 7: Size dependence of the chirality of the lowest state in each subspace of the Heisenberg model which is estimated through $\sqrt{3\left\langle\left(Q^{z}\right)^{2}\right\rangle} / N$. The data for $N=36$ is also listed 122 . SW denotes the result from the spin-wave expansion $\mathrm{B}^{\mathrm{G}}$.

Figure 8: Spontaneous symmetry breaking of the chirality of the $X Y$ model which is created by a linear combination $\frac{1}{\sqrt{2}}(|\alpha\rangle+i|\beta\rangle)$ of the low-lying state of the type $\alpha$ and that of $\beta$.

Figure 9: Spontaneous symmetry breaking of the chirality of the Heisenberg model which is created by a linear combination $\frac{1}{\sqrt{2}}(|\alpha\rangle+i|\beta\rangle)$ of the low-lying state of the type $\alpha$ and that of $\beta$. The values are multiplied by the factor $\sqrt{3}$. 


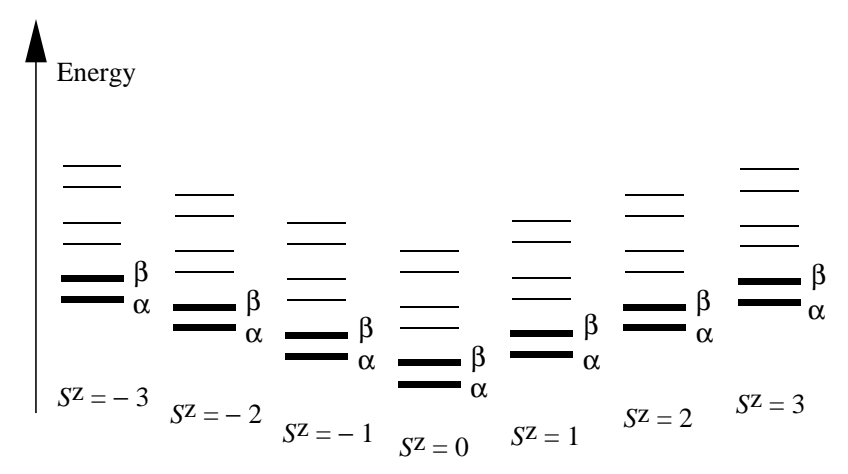

Fig. 1

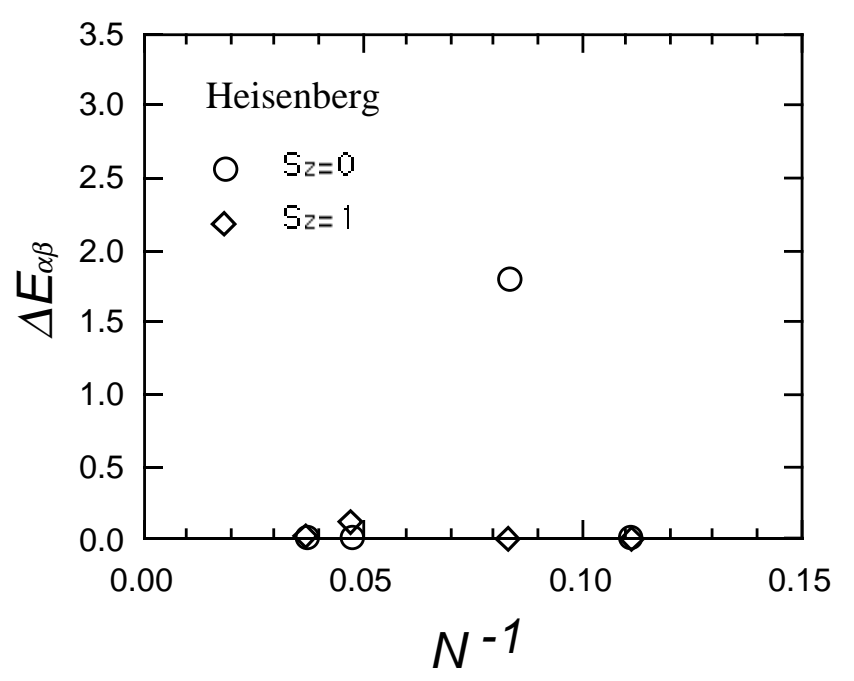

Fig. 2

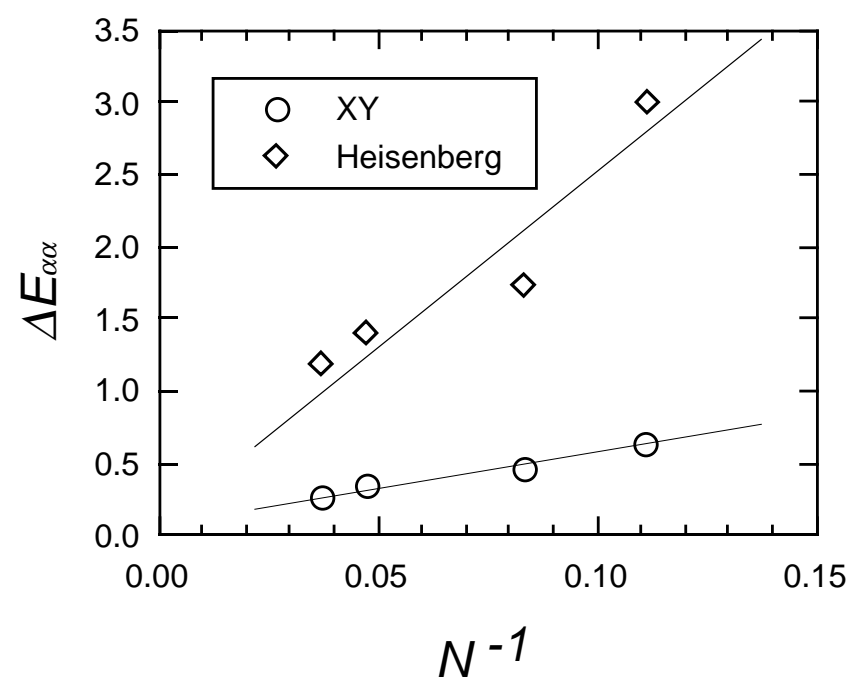

Fig. 3 


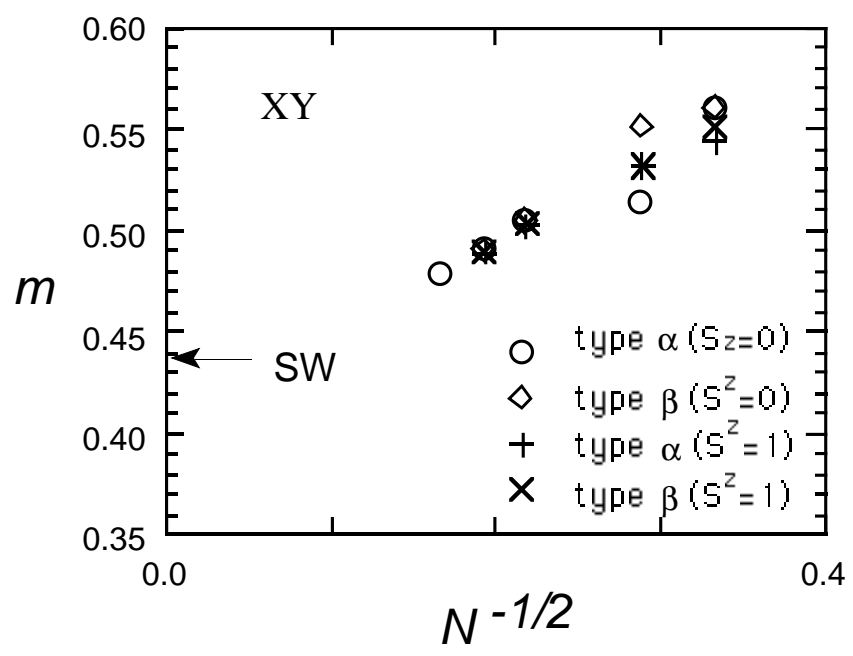

Fig. 4

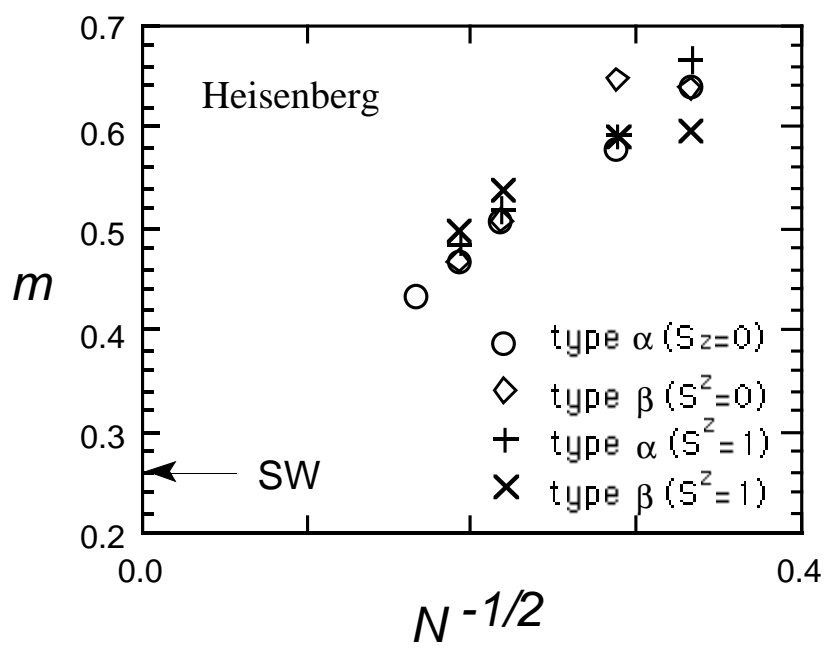

Fig. 5

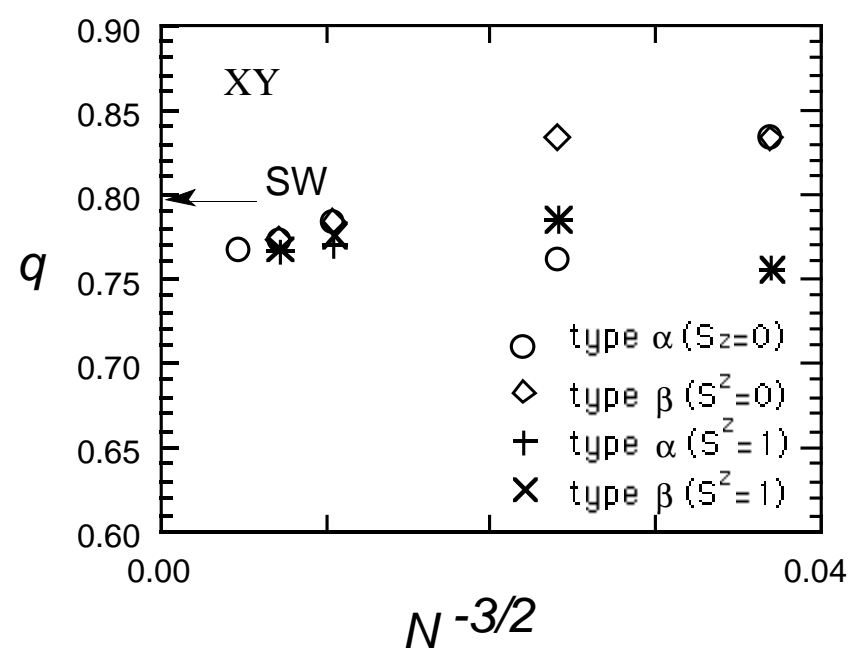

Fig. 6 


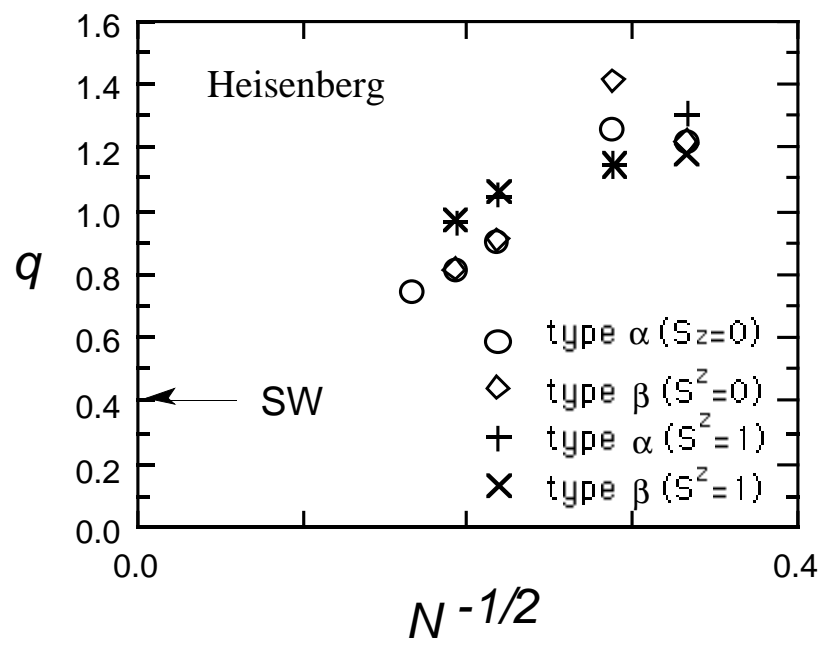

Fig. 7

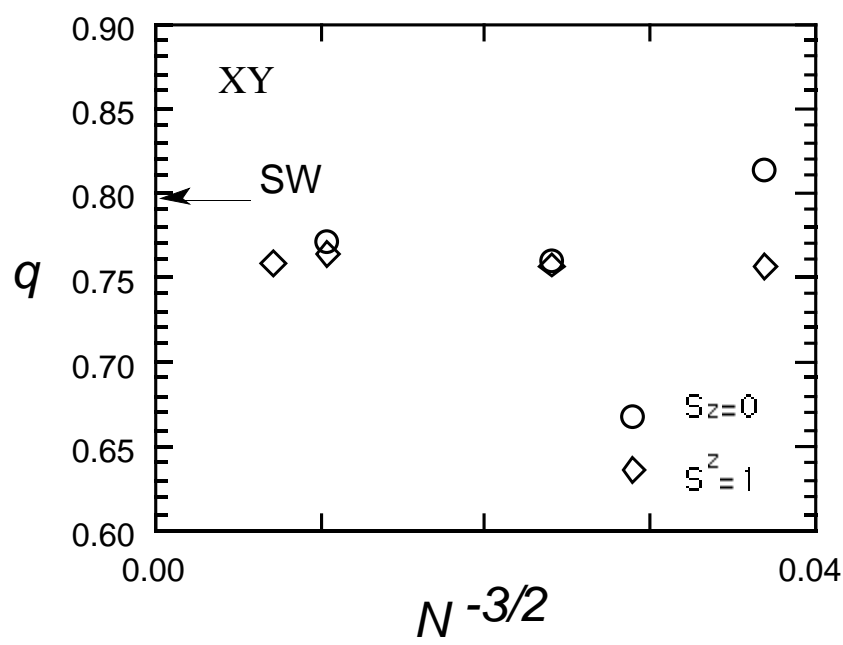

Fig. 8

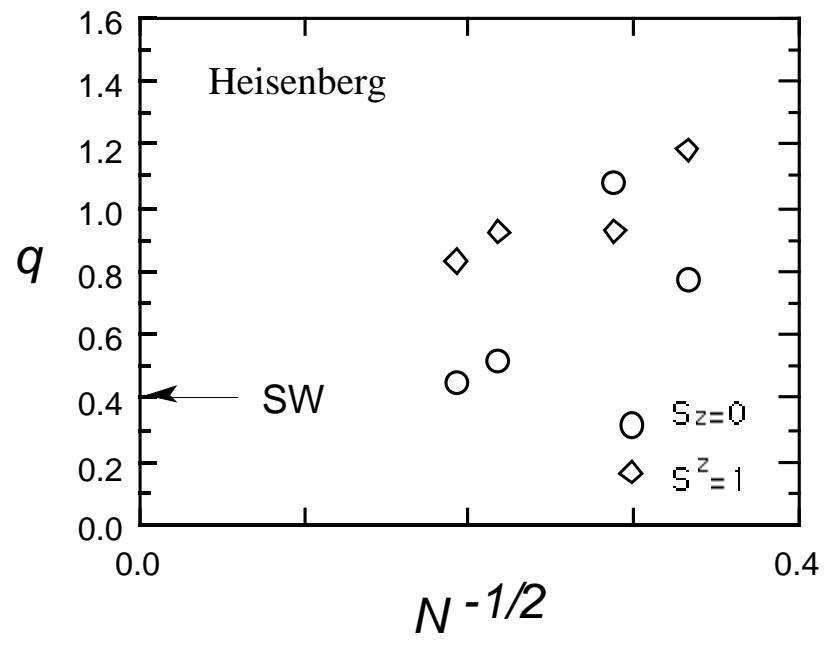

Fig. 9 